\title{
Laparoscopic Subtotal Cholecystectomy for Difficult Acute Calculous Cholecystitis
}

\author{
Hamdy Sedky Abdallah \\ General Surgery Department, Faculty of Medicine, Tanta University, Tanta, Egypt
}

Email address:

Hamdy.abdallah@med.tanta.edu.eg, hamdysedky@hotmail.com

\section{To cite this article:}

Hamdy Sedky Abdallah. Laparoscopic Subtotal Cholecystectomy for Difficult Acute Calculous Cholecystitis. Journal of Surgery. Vol. 5, No. 6, 2017, pp. 111-117. doi: 10.11648/j.js.20170506.15

Received: September 29, 2017; Accepted: October 10, 2017; Published: November 12, 2017

\begin{abstract}
Background: When the critical view of safety (CVS) can't be obtained during dissection of Calot's triangle in difficult gallbladder, conversion to open surgery or other "damage control" alternatives as cholecystostomy and subtotal cholecystectomy are recommended to prevent bile duct injury. Materials and methods: The medical records of all patients presented with acute calculous cholecystitis (ACC) during the study period were retrospectively reviewed and analyzed. Results: Laparoscopic cholecystectomy (LC) was attempted in 71 difficult gallbladders out of 379 patients presenting with ACC. In 6 patients $(8.5 \%)$, conversion to open surgery or laparoscopic cholecystostomy was performed. Laparoscopic subtotal cholecystectomy (LSC) with dissection and control of the cystic duct was performed for the remaining 65 patients $(91.5 \%)$ including 50 females (77\%) and 15 males (23\%) with a mean age of $42.35 \pm 12.4$ years. The mean operative blood loss was $45.28 \pm 18.6 \mathrm{CC}$ and the mean operative time was $96.3 \pm 24.19$ minutes. There were no operative complications or mortality. The mean hospital stay was $28 \pm 17.8$ hours. There was no postoperative jaundice, bile leak, intra-abdominal collections or mortality. Conclusion: When surgery is indicated for difficult ACC, LSC with control of the cystic duct is safe with excellent outcomes. However, if the CVS can't be achieved due to obscured anatomy at Calot's triangle, conversion to open surgery or cholecystostomy must be performed to prevent bile duct injury.
\end{abstract}

Keywords: Subtotal Cholecystectomy, Laparoscopy, Acute Calculous Cholecystitis

\section{Introduction}

Cholelithiasis affects about 20 million people in the United States yearly and $20 \%$ of symptomatic patients will develop acute calculous cholecystitis (ACC) [1]. Laparoscopic cholecystectomy (LC) was initially considered unsafe and harmful for ACC, but currently, it is the most commonly performed procedure in this setting [2].

Several studies had confirmed the safety of LC for ACC performed within the first few days of the disease onset $[3,4$, 5]. For late presenting patients, most surgeons find LC too difficult and risky, preferring a conservative treatment followed by delayed LC, a few weeks later, after resolution of the acute episode. However, up to $30 \%$ of these patients require subsequent cholecystectomy before the scheduled date because of either failed response to conservative treatment or recurrent episodes of inflammation [6,7].

With an incidence of $16 \%$ in a large series, difficult gallbladder (GB) is usually associated with severe inflammation that distorts the local anatomy and renders dissections more difficult and risky (ACC, empyema, gangrene, perforation, and Mirizzi syndrome) or with liver cirrhosis that increases the risk of bleeding [8]. When the critical view of safety (CVS) can't be obtained during dissection of Calot's triangle in difficult GB, conversion to open surgery or other "damage control" alternatives such as cholecystostomy, fundus-first approach and subtotal cholecystectomy (SC), are recommended to decrease the risks related to difficult GB especially common bile duct (CBD) injury $[2,9,10]$. Conversion per se, however, does not always provide a better view of the obscured anatomy, and for those with less experience using the open approach, conversion may fail to prevent bile duct injury [11].

SC involves removal of a portion of the GB wall, with or without closure of the Hartmann's pouch or cystic duct (CD), when the CVS cannot be achieved [12]. The aim of this study 
was to retrospectively report my experience of laparoscopic $\mathrm{SC}$ for difficult ACC including the intraoperative decision making, surgical technique and outcomes.

\section{Materials and Methods}

\subsection{Study Design}

This retrospective case series was conducted at the Gastrointestinal and Laparoscopic Surgery Unit, General Surgery Department, Tanta University, Tanta, Egypt. The medical records of all patients presented with ACC during the period from June 2008 to August 2016 were reviewed. Patients who had standard LC (SLC) for uncomplicated GB stones or ACC were excluded. Preoperative data including history, findings of physical examination, preoperative investigations were retrieved. The operative findings including details of the operative technique, complications, estimated blood loss, operative time and conversions were obtained. The postoperative data including the hospital stay and any postoperative complications, readmission, interventions and mortality were collected. Primary end-points were the conversion to open surgery or the occurrence of bile duct injury and secondary end-points were the other intraoperative and the postoperative complications. All patients had already been consented prospectively and the study protocol was approved by the "Research Ethics Committee" of the Faculty of Medicine, Tanta University, Tanta, Egypt.

\subsection{Statistical Analysis}

Categorical data were expressed as frequencies while metric data were expressed as range, mean, standard deviation (SD) and median using the Microsoft Excel 2013 software.

\subsection{Operative Technique}

Surgery was performed by the author who has about 20 years of experience in laparoscopic surgery. After induction of the pneumoperitoneum and insertion of the standard laparoscopic ports, exploration of the abdominal cavity was performed. Adherent omentum and bowel were dissected off the GB and any peri-cholecystic abscess was aspirated and sampled for bacteriologic studies. The GB was aspirated via a Veress needle or through a perforation made at the fundus. The GB fundus was then grasped and retracted. At this point, a decision was to be made regarding the next step Figure 1. If inflammation and adhesions at Calot's triangle prevents safe dissection of the Hartmann's pouch or CD (impossible Calot), then, either a conversion to open surgery or laparoscopic cholecystostomy through the hole at the fundus was performed. If the local conditions permit, dissection exposes the Hartmann's pouch which was grasped and retracted upwards, sometimes after milking of the impacted stone. The Hartmann's pouch and/or CD were dissected, then, closed using intra-corporeal stiches, sutures or surgical clips. Intraoperative cholangiogram was used when deemed necessary. Now, another decision was necessary; whether to proceed to SLC or LSC Figure 1.

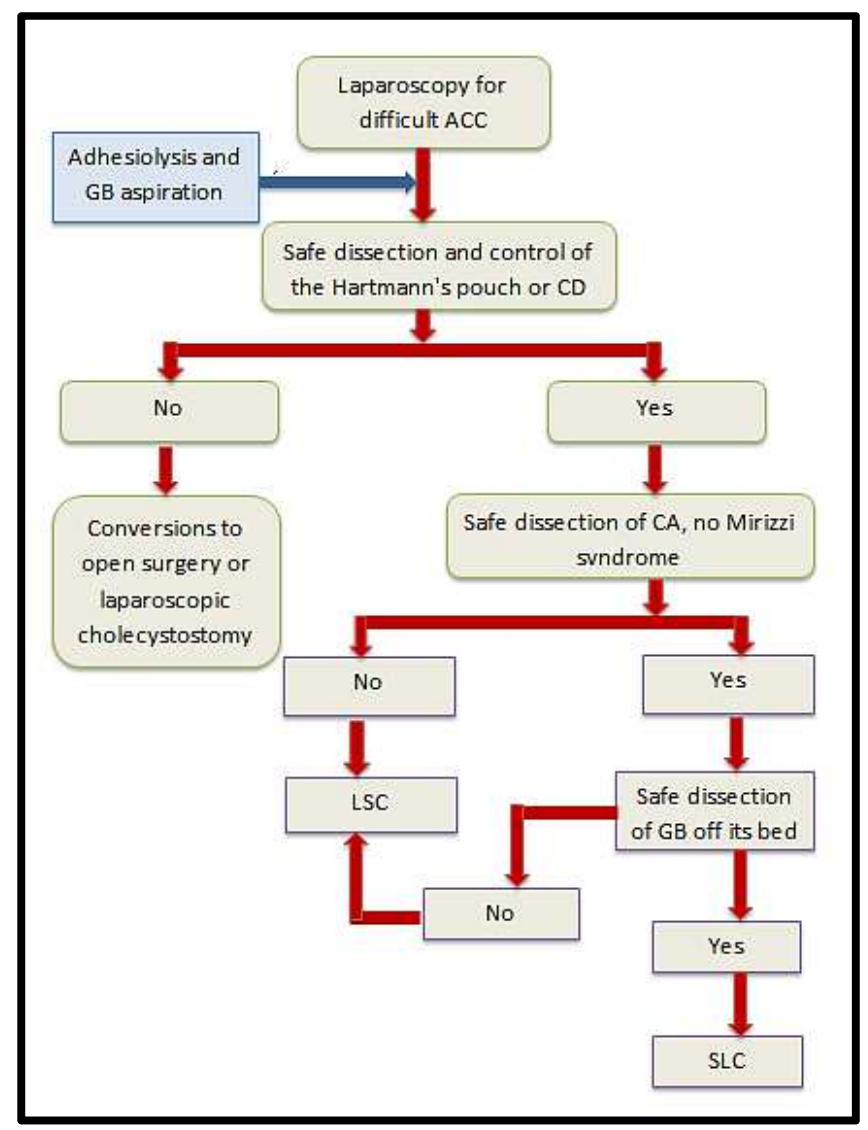

Figure 1. A Flow Chart Showing the Intra-Operative Decision Making. ACC: Acute Calculous Cholecystitis, GB: Gallbladder, CA: Cystic duct, CD: Cystic artery, LSC: Laparoscopic Subtotal Cholecystectomy, SLC: Standard Laparoscopic Cholecystectomy.

SLC was chosen if it was possible to safely dissect the cystic artery in Calot's triangle and the GB off its bed. If safe dissection of these two structures was not possible, LSC was chosen Figure 2. Starting at the Hartmann's pouch and using the hook or the Harmonic Ultrasound Shear, the anterior wall of the GB was cut all around 1-2 mm away from the liver leaving the posterior wall of the GB in place while evacuating stones into an Endo-bag as soon as they appear. The excised GB wall was also evacuated into the Endo-bag. Haemostasis was assured; the remnant GB mucosa was coagulated with diathermy, the surgical field was irrigated well with saline and a drain was left in the Morrison's pouch when needed. 


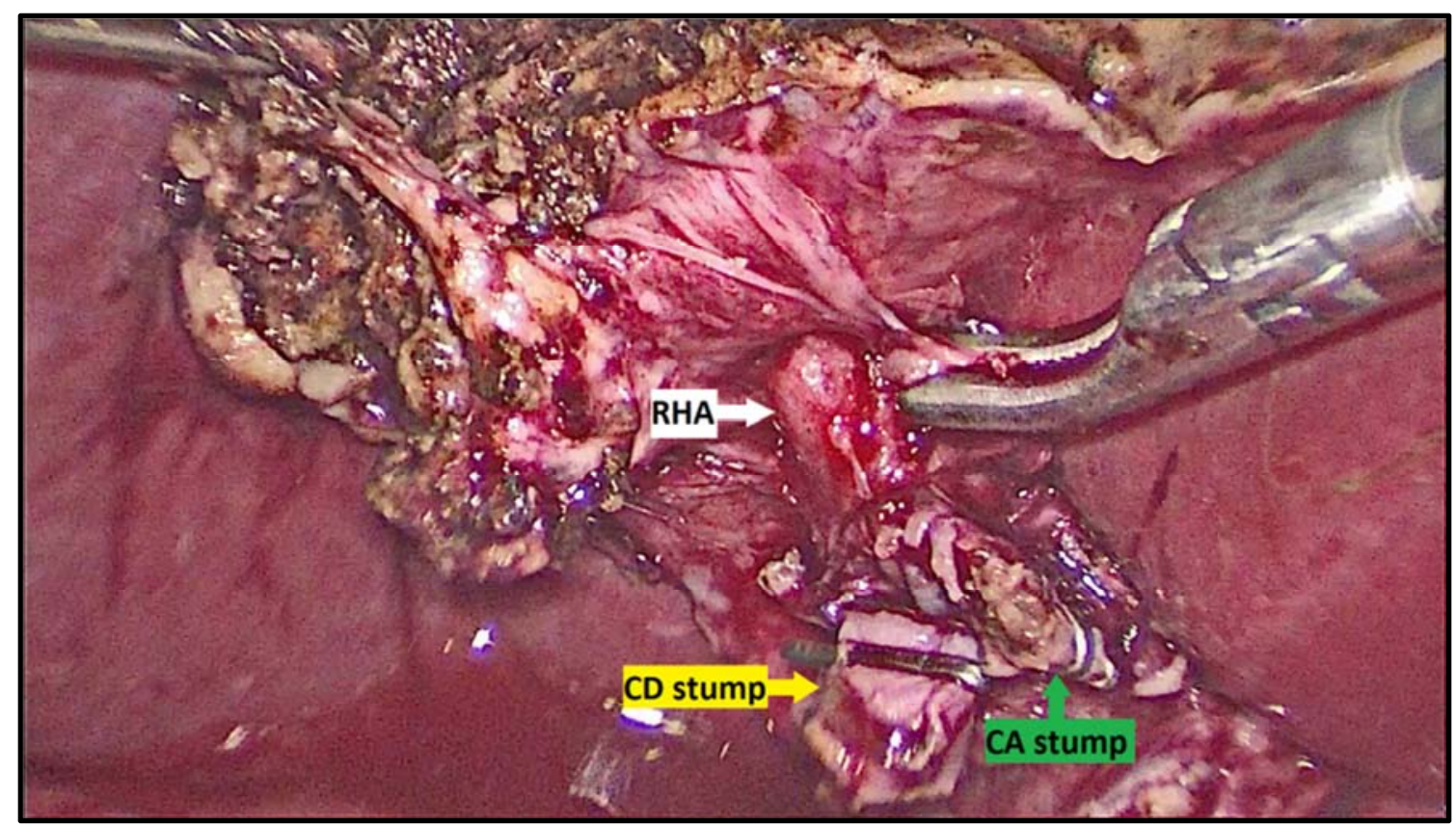

Figure 2. The RHA is Entering the Gallbladder Bed. Since Resection of the Posterior Gallbladder Wall may Injure This Artery, Laparoscopic Subtotal Cholecystectomy was Performed. RHA: Right Hepatic Artery, CD: Cystic Duct Stump, CA: Cystic Artery Stump.

In patients with Mirizzi syndrome type I [Figure 3], no attempt was made to dissect the adherent Hartmann's pouch off the extrahepatic bile ducts. In these patients, LSC was performed when it was possible to safely dissect the CD, otherwise, conversion to open surgery was performed.

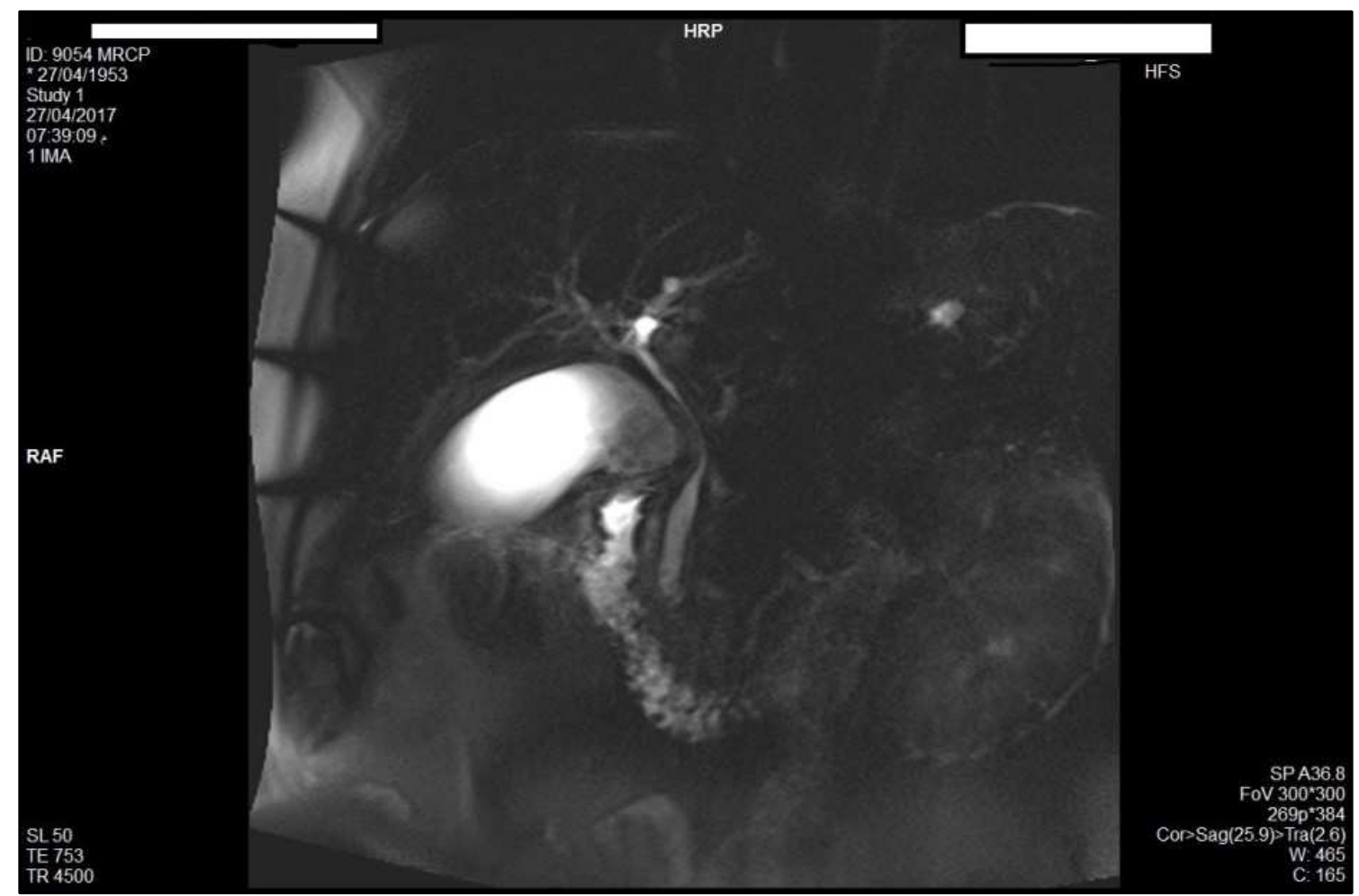

Figure 3. Magnetic Resonance Cholangiopancreatography Showing Mirizzi Syndrome. Since Resection of the Hartmann's Pouch may Injure the Common Hepatic Duct, Laparoscopic Subtotal Cholecystectomy was Performed.

\section{Results}

During the study period, 379 patients presented with ACC.
Figure 4 Three hundred three patients presented within 7 days of the disease onset; all had SLC and were excluded from the analysis. 


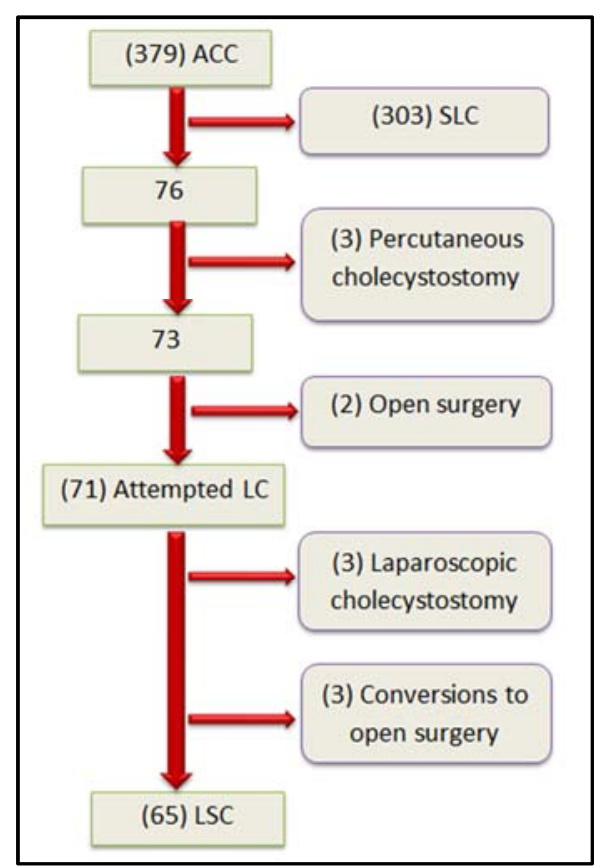

Figure 4. Patients' Population Stratified According to Type of Surgery. ACC: Acute Calculous Cholecystitis, SLC: Standard Laparoscopic Cholecystectomy, LC: Laparoscopic Cholecystectomy, LSC: Laparoscopic Subtotal Cholecystectomy.

Three elderly patients with multiple comorbidities were considered poor surgical candidates and were referred for percutaneous cholecystostomy. Two other patients were submitted to open surgery as they were considered contraindications for laparoscopy (Cardio-respiratory disease in 1 and previous upper abdominal surgery in the other) and were also excluded. LC was attempted in 71 patients, all presenting $>7$ days after the disease onset. In 6 of them, conversion to open surgery $(3 / 71 ; 4.2 \%)$ or laparoscopic cholecystostomy $(3 / 71 ; 4.2 \%)$ was performed because the anatomy at Calot's triangle was obscured by inflammation and adhesions making safe dissection of the Hartmann's pouch and CD impossible. The remaining 65 patients had LSC and were included in the final analysis. Of the 2 patients with Mirizzi syndrome, 1 was converted to open surgery and the other had LSC after dissection and control of the CD.

\subsection{Preoperative Results}

The study population included 50 females (77\%) and 15 males $(23 \%)$ with a mean age of $42.35 \pm 12.4$ years (range 23-67, median 44). Twenty-one patients (32.3\%) had hypertension, 17 (26\%) had diabetes mellitus (DM), 15 (23\%) were $\mathrm{HCV}+$ ve and $11(17 \%)$ had compensated liver cirrhosis Table 1.

The presenting symptoms included abdominal pain, vomiting and fever. The duration of symptoms ranged from 11 to 45 days with a mean of $27.5 \pm 13.3$ days (median 24) days before surgery. Nine patients $(13.8 \%)$ had endoscopic retrograde cholangiography (ERC) for clearance of CBD stones 2 weeks before surgery. Two patients (3.1\%) had resolving acute pancreatitis; 1 on medical treatment and the other after ERC and extraction of an ampullary stone.
Physical examination revealed a palpable right hypochondrial masses in $16(24.6 \%)$ patients. Laboratory tests suggestive of ACC included elevated white blood cell (WBC) count and \pm ve C-reactive protein (CRP) level. Serum bilirubin was elevated in 4 patients (6.2\%). Abdominal ultrasonography (US) showed distended thick-walled GB in all patients, a stone impacted at the neck of the GB in 52 patients $(80 \%)$ with pericholecystic fluid collection in 6 patients $(9.2 \%)$.

Table 1. Pre-Operative Results.

\begin{tabular}{|c|c|c|}
\hline Variable & No of patients (65) & $(\%)$ \\
\hline \multicolumn{3}{|l|}{ Sex } \\
\hline Female & 50 & 77 \\
\hline Male & 15 & 23 \\
\hline \multicolumn{3}{|l|}{ Age (years) } \\
\hline Range & $23-67$ & \\
\hline Mean & $42.35+12.4$ & \\
\hline Median & 44 & \\
\hline \multicolumn{3}{|l|}{ Co-Morbidities } \\
\hline Hypertension & 21 & 32.3 \\
\hline $\mathrm{DM}$ & 17 & 26 \\
\hline $\mathrm{HCV}+\mathrm{ve}$ infection & 15 & 23 \\
\hline Compensated liver cirrhosis & 11 & 17 \\
\hline \multicolumn{3}{|l|}{ Duration of symptoms (days) } \\
\hline Range & $11-45$ & \\
\hline Mean & $27.5+13.3$ & \\
\hline Median & 24 & \\
\hline Previous ERC & 9 & 13.8 \\
\hline Resolving acute pancreatitis & 2 & 3.1 \\
\hline Palpable abdominal masses & 16 & 24.6 \\
\hline \multicolumn{3}{|l|}{ Laboratory tests } \\
\hline Elevated $\mathrm{WBC}$ and +ve CRP & 47 & 72.3 \\
\hline Elevated serum bilirubin & 4 & 6.2 \\
\hline \multicolumn{3}{|l|}{ US finding } \\
\hline Stone impacted at GB neck & 52 & 80 \\
\hline Pericholecystic abscess & 6 & 9.2 \\
\hline \multicolumn{3}{|l|}{ Obstructive jaundice } \\
\hline Mirizzi syndrome type I & 2 & 3.1 \\
\hline $\mathrm{CBD}$ stone & 2 & 3.1 \\
\hline \multicolumn{3}{|l|}{ Indication of surgery } \\
\hline Failure of medical treatment & 47 & 72.3 \\
\hline Recurrent ACC & 9 & 13.8 \\
\hline Pericholecystic abscess & 6 & 9.2 \\
\hline Obstructive jaundice & 4 & 6.2 \\
\hline
\end{tabular}

DM: diabetes mellitus, $\mathrm{HCV}$ : hepatitis $\mathrm{C}$ virus, ERC: endoscopic retrograde cholangiography, WBC: white blood cell, CRP: C-reactive protein, GB: gallbladder, CBD: common bile duct, ACC: Acute calculous cholecystitis.

MRCP showed evidence of biliary obstruction due to either CBD stones in 2 patients (3.1\%) or Mirizzi syndrome type I in 2 other patients (3.1\%). Figure 3 All the patients had moderate (grade II) ACC according to Tokyo grading 2007 [13]. The indications of surgery were persistent biliary pain not responding to medical treatment in 47 patients $(72.3 \%)$, recurrent ACC after initial improvement in 9 patients (13.8\%), ACC with pericholecystic abscess in 6 patients $(9.2 \%)$ and ACC with obstructive jaundice in 4 patients $(6.2 \%)$.

\subsection{Operative Results}

The GB wall had patchy necrosis in 9 patients $(13.8 \%)$ and was perforated in $5(7.7 \%)$ of them. Six patients $(9.3 \%)$ had pericholecystic abscesses. Table 2 . 
Table 2. Operative Results.

\begin{tabular}{|c|c|c|}
\hline Variable & No of patients (65) & $(\%)$ \\
\hline \multicolumn{3}{|l|}{ Operative findings } \\
\hline Patchy necrosis of GB wall & 9 & 13.8 \\
\hline Perforated GB & 5 & 7.7 \\
\hline Pericholecystic abscess & 6 & 9.3 \\
\hline Intraoperative cholangiogram & 3 & 4.6 \\
\hline Intraoperative ERC & 2 & 3.1 \\
\hline Peritoneal drain & 42 & 64.6 \\
\hline \multicolumn{3}{|l|}{ Operative blood loss (ml) } \\
\hline Range & 20-105 & \\
\hline Mean & $45.28+18.6$ & \\
\hline Median & 43 & \\
\hline \multicolumn{3}{|l|}{ Operative time $(\mathrm{min})$} \\
\hline Range & $65-155$ & \\
\hline Mean & $96.3+24.19$ & \\
\hline Median & 94 & \\
\hline Operative complications & 0 & 0 \\
\hline Operative mortality & 0 & 0 \\
\hline
\end{tabular}

GB: gallbladder, ERC: endoscopic retrograde cholangiography.

It was possible to safely dissect, suture, ligate or clip the Hartmann's pouch and/or the $\mathrm{CD}$ in all patients. Intraoperative cholangiogram was needed in 3 patients $(4.6 \%)$. A drain was inserted in 42 patients $(64.6 \%)$. The operative blood loss varied from 20 to $105 \mathrm{CC}$ with a mean of $45.28 \pm 18.6 \mathrm{CC}$ (median $43 \mathrm{CC}$ ) and the operative time varied from 65 to 155 minutes with a mean of $96.3 \pm 24.19$ minutes (median 94). Two patients with $\mathrm{CBD}$ stones had ERC for clearance of the CBD under the same anaesthesia. There were no operative complications or mortality.

\subsection{Postoperative Results}

The drain was removed 24 hours after surgery in 31 patients $(68.6 \%)$, and after 72 hours in the remaining 11 patients (31.4\%). Three patients $(4.6 \%)$ had superficial port site infection and 1 patient $(1.5 \%)$ had post-ERC bleeding that was controlled endoscopically. The postoperative hospital stay ranged from 24-72 hours with a mean of $28 \pm 17.8$ hours (median 24 hours). No patients developed postoperative jaundice, bile leak or intra-abdominal collections. Only 1 patient needed readmission for control of post-ERC bleeding. There was no postoperative mortality. Pathologic examination confirmed the diagnosis of ACC in all patients. Sixty patients $(92.3 \%)$ were followed-up for 6 months postoperatively and all were symptom-free with normal liver function tests and US data at 6 months. Table 3.

Table 3. Postoperative Results.

\begin{tabular}{|c|c|c|}
\hline Variable & No of patients (65) & $(\%)$ \\
\hline \multicolumn{3}{|l|}{ ICU admission } \\
\hline No of patients & $3(4.6 \%)$ & \\
\hline Mean duration (hours) & $19.3 .1+4.16$ & \\
\hline Median duration (hours) & 18 & \\
\hline \multicolumn{3}{|l|}{ Duration of abdominal drainage (hours) } \\
\hline 24 & 31 & 8.6 \\
\hline 72 & 11 & 31.4 \\
\hline \multicolumn{3}{|l|}{ Post-operative complications } \\
\hline Superficial port site infection & 3 & 4.6 \\
\hline Post-ERC Bleeding & 1 & 1.5 \\
\hline
\end{tabular}

\begin{tabular}{lcc}
\hline Variable & No of patients (65) & (\%) \\
\hline Jaundice & 0 & 0 \\
Bile leak & 0 & 0 \\
Intra-abdominal collection & 0 & 0 \\
Post-operative hospital stay (hours) & \multicolumn{2}{c}{$24-72$} \\
Range & \multicolumn{2}{c}{$28+17.6$} \\
Mean & \multicolumn{2}{c}{24} \\
Median & 1 & 1.5 \\
Postoperative interventions & 0 & 0 \\
Postoperative mortality & \multicolumn{2}{c}{} \\
\hline
\end{tabular}

ICU: intensive care unit, ERC: endoscopic retrograde cholangiography.

\section{Discussion}

Patients with ACC presenting $72 \mathrm{~h}$ or more after the onset of symptoms are usually treated conservatively and subsequently readmitted for delayed LC 6-12 weeks later. About $25-29 \%$ of these patients require surgery before their scheduled delayed cholecystectomy because of treatment failure, recurrent attack of ACC or development of complications $[6,7,14,15]$. In these patients, inflammatory reactions including congestion, oedema, induration and adhesions at Calot's triangle make laparoscopic dissection extremely difficult in a significant proportion of patients, thus, increasing the morbidity related to bile duct injury [14]. To prevent this catastrophe, a difficult LC conventionally involves conversion to open surgery with the subsequent loss of the advantages of laparoscopy. Even after conversion, cholecystostomy is sometimes all what can be done, because of severe inflammation and unclear anatomy [16-18]. In these difficult scenarios, alternative surgical techniques and procedures including LSC or cholecystostomy are usually needed to prevent the catastrophic complication of major biliary or vascular injury and to decrease the possibility of conversion.

The main outcome of this study shows that LSC performed for difficult ACC proved to be a safe way out of the dilemma. It prevented the serious complication of bile duct injury and the excessive bleeding from the GB bed $(17 \%$ of patients in this study had liver cirrhosis). The dissection and control of the CD prevented the complications of postoperative bile leak and the consequent therapeutic interventions sometimes needed to control it. However, when it was not possible to safely identify and dissect the Hartmann's pouch and/or CD to achieve the CVS, attempts at further dissection stopped and conversion to open surgery or cholecystostomy was performed to prevent bile duct injury.

There is no standardized technique of SC in literature. In a systematic review published in 2013, Henneman et al, identified 4 different techniques of SC in literature. The first 2 involve excision of most of the GB anterior wall, leaving the posterior wall attached to the liver and the remaining GB stump is closed or not. The other 2 techniques include partial resection of both the anterior and posterior GB wall and transection of the GB at the Hartmann's pouch, with or without closure of the remnant GB pouch left behind. Irrespective of the technique used, the Hartmann's pouch and/or CD were closed in $53 \%$ of patients only and a drain 
was used routinely when the CD was left open [12]. In this review, the median operative time was $81.1 \mathrm{~min}$, the conversion rate was $10.4 \%$, and the median hospital stay was 4.5 days. Bile duct injury occurred in $1 / 60(1.7 \%)$ of patients. Postoperative bile leak developed in $5.6 \%$ of patients with a closed CD compared with $16 \%$ with an open CD. Four patients $(2.2 \%)$ had recurrent symptomatic GB stone; 3 were managed with endoscopic papillotomy and 1 required re-surgery. Postoperative ERC to manage biliary leakage from the CD stump was needed for $2.7 \%$ of patients when the CD was closed compared with $16 \%$ when the CD was left open. In $1.5 \%$ (all had open CD), a percutaneous intervention was needed because of subhepatic or subphrenic abscess or hematoma. Reoperation was performed for 8 of 292 patients $(2.7 \%) ; 3$ for intra-abdominal abscess, 2 for persistent bile leak, 1 for removal of an infected residual stone and 1 for bleeding from the liver bed. [12].

A newer systematic review and meta-analysis of SC was published in JAMA Surgery 2016 that included 1231 patients $73 \%$ had LSC, $17 \%$ had open SC. The CD or GB stump was closed in $91.4 \%$ and left open in $8.6 \%$ of patients. In this review, bile leak developed in $42 \%$ of patients in whom the CD was left open and in $16.5 \%$ following closure of the CD and this difference was found statistically significant. Similarly, patients in whom the CD was left open, in comparison to those who had the CD closed, had higher incidences of sub-phrenic collection (2 needed radiologic drainage and an 1 underwent a re-operation), retained stones (all needed endoscopic or surgical treatment), reoperations for various indications and 30-day mortality but, the differences between the 2 subgroups were statistically insignificant [19].

In fact, the decision making in this challenging situation is quite difficult because each of the available alternatives; conversion to open surgery, cholecystostomy and SC as described in literature has its own disadvantages. For conversion, the disadvantages are obvious: loss of the advantages of laparoscopy as a minimal access surgery. Patients having cholecystostomy will need a re-admission, later on, for definitive surgery. SC, as described in literature, is attended with a long list of complications that may need a $2^{\text {nd }}$ surgical, endoscopic or radiologic intervention.

Contrary to the published techniques of SC, I dissected and closed the $\mathrm{CD}$ as long as this was safely possible. I believe that closure of the CD during LSC, as described in this study, was responsible for the better results, in comparison to the published results, in terms of bile leak, intra-abdominal collection, the need for postoperative ERC, recurrent or retained GB stones, intervention, re-surgery, hospital stay and 30-day mortality. When safe dissection of the CD was not possible due to unclear anatomy at Calot's triangle, I converted to open surgery or performed cholecystostomy. Again, I believe that conversion to open surgery or performing cholecystostomy prevented bile duct injury in this study.

Only the operative time was longer in this study in comparison to that reported by Henneman et al [12]. This difference is expected and is due to the time needed to dissect and control the CD in this study.

Despite the good results obtained in this study, this approach has 2 important drawbacks. The $1^{\text {st }}$ is that the part of the GB left behind may, theoretically, harbor malignant foci although the risk is small; GB cancer is reportedly found in $0.2-0.8 \%$ of patients undergoing LC for symptomatic GB stones $[20,21]$. The $2^{\text {nd }}$ is the longer operative time in relation to published results. Also, it is important to be noted that the good results reported in this study were achieved by a surgeon with a considerable experience in laparoscopic surgery, implying that similar results may not always be achievable by surgeons of different skills and experience. This study has some limitations; the retrospective nature and the small sample size are the main limitation of the study.

\section{Conclusion}

When surgery is indicated for difficult ACC, LSC with control of the $\mathrm{CD}$ performed by an experienced surgeon is safe with excellent outcomes. It is associated with a high success rate, low PO complication rate and short PO hospital stay. However, if the CVS can't be achieved due to obscured anatomy at the Calot's triangle, conversion to open surgery or Cholecystostomy must be performed to prevent bile duct injury.

\section{Conflict of Interest Statement}

The author declares that he has no competing interests.

\section{References}

[1] Strasberg SM (2008) Acute calculous cholecystitis. N Engl J Med 358:2804-11.

[2] Buddingh KT, Hofker HS, ten Cate Hoedemaker HO, et al (2011) Safety measures during cholecystectomy: results of a nationwide survey. World J Surg 35:1235-1241.

[3] Bender JS, Zenilman ME (1995) Immediate laparoscopic cholecystectomy as definitive therapy for acute cholecystitis. Surg Endosc 9: 1081-1084.

[4] Garber SM, Korman J, Cosgrove JM, Cohen JR (1997) Early laparoscopic cholecystectomy for acute cholecystitis. Surg Endosc 11: 347-350.

[5] Koo KP, Thirlby RC (1996) Laparoscopic cholecystectomy in acute cholecystitis: what is the optimal time for operation? Arch Surg 131: 540-545

[6] Lo CM, Liu CL, Lai ECS, Fan ST, Wong J (1996) Early versus delayed laparoscopic cholecystectomy for treatment of acute cholecystitis. Ann Surg 223: 37-42.

[7] McArthur P, Cuschieri A, Sells A, Shields R (1975) Controlled clinical trial comparing early with interval cholecystectomy for acute cholecystitis. Br J Surg 62: 850-852.

[8] Wolf AS, Nijsse BA, Sokal SM, et al (2009) Surgical outcomes of open cholecystectomy in the laparoscopic era. Am J Surg 197:781-784. 
[9] Lee J, Miller P, Kermani R, Dao H, O' Donnell K (2012) Gallbladder damage control: compromised procedure for compromised patients. Surg Endosc 26(10):2779-2783.

[10] Eikermann M, Siegel R, Broeders I, et al (2012) Prevention and treatment of bile duct injuries during laparoscopic cholecystectomy: the clinical practice guidelines of the European Association for Endoscopic Surgery (EAES). Surg Endosc 26 (11):3003-3039.

[11] Booij KA, de Reuver PR, van Delden OM, Gouma DJ (2009) Conversion has to be learned: bile duct injury following conversion to open cholecystectomy. Ned Tijdschr Geneeskd 153:A296.

[12] Henneman D, da Costa DW, Vrouenraets BC, et al (2013) Laparoscopic partial cholecystectomy for the difficult gallbladder: a systematic review. Surg Endosc 27(2):351-358.

[13] Hirota M, Takada T, Kawarada Y, et al (2007) Diagnostic criteria and severity assessment of acute cholecystitis: Tokyo Guidelines J Hepatobiliary Pancreat Surg 14:78-8.

[14] Tzovaras G, Zacharoulis D, Liakou P, et al (2006) Timing of laparoscopic cholecystectomy for acute cholecystitis: a prospective nonrandomized study. World J Gastroenterol 12:5528-5531.
[15] Mercer SJ, Knight JS, Toh SK, et al (2004) Implementation of a specialist-led service for the management of acute gallstone disease. Br J Surg 91:504-508.

[16] Rattner DW, Ferguson C, Warshaw AL (1993) Factors associated with successful laparoscopic cholecystectomy for acute cholecystitis. Ann Surg 217: 233-6.

[17] Schafer M, Krahenbuhl L, Buchler MW (2001) Predictive factors for the type of surgery in acute cholecystitis. Am J Surg 182: $291-7$.

[18] Livingston EH, Rege R (2004) A nationwide study of conversion from laparoscopic to open cholecystectomy. Am J Surg 188: 205-11.

[19] Elshaer M, Gravante G, Thomas K, et al (2015) Subtotal Cholecystectomy for "Difficult Gallbladders" Systematic Review and Meta-analysis JAMA Surg 150 (2):159-168.

[20] Yamamoto H, Hayakawa N, Kitagawa Yet al (2005) Unsuspected gallbladder carcinoma after laparoscopic cholecystectomy. J Hepatobillary Pancreat Surg 12:391-8.

[21] Ouchi K, Mikuni J, Kakugawa Y, Organizing Committee of the 30th Annual Congress of the Japanese Society of Biliary Surgery (2002) Laparoscopic cholecystectomy for gallbladder carcinoma: results of a Japanese survey of 498 patients. J Hepatobiliary Pancreat Surg 9:256-60. 